\title{
Evaluación de ancho de banda en transmisiones multicast sparse mode implementadas en redes IPV6
}

\section{Evaluation of bandwidth in sparse mode multicast transmissions implemented in IPV6 networks}

\author{
Pineda Ancco, Ferdinand ${ }^{\mathrm{a}}$; Cruz de la Cruz, Joséb; Mamani Pari, David ${ }^{\mathrm{c}}$ \\ a, bUniversidad Nacional del Altiplano, Puno, Perú \\ 'Universidad Peruana Unión \\ Recibido el 10 de agosto del 2015 - Aceptado el 20 de noviembre del 2015
}

\begin{abstract}
Resumen
En el trabajo de investigación se describió el diseño e implementación del protocolo multicast de tipo Sparse mode con el objetivo de mejorar el tráfico de datos en redes que transporten información unidireccional. El diseño se realiza sobre una red que implementa IPV6 como protocolo de capa 3 del modelo de referencia OSI. Para validar el método, la evaluación del tráfico se realizó analizando el ancho de banda, retardos y cantidad de bits enviados por el protocolo multicast Sparse mode cursado en routers que utilizan IPV6 e IPV4 en modo individual o en Dual Stack. Asimismo, para el análisis se utilizó Jperf realizando pruebas con diversos parámetros. Se implementó una red piloto con 5 routers utilizando OSPF como protocolo de enrutamiento dinámico unicast. Como base para la implementación de multicast Sparse mode, se utilizó también dos enlaces inalámbricos entre 4 nodos de la red para conocer la respuesta de multicast en dos escenarios. Para la validación se realizó 10 transmisiones bajo una red IPV4, IPV6 (escenario 1), y Dual stack (escenario 2), tomándose los datos promedio de cada transmisión y conocer el ancho de banda, retardos y cantidad de datos transmitidos. Esta implementación se realizó en el laboratorio CISCO de la Universidad Nacional del Altiplano.

Como hipótesis se considera que emisiones multicast Sparse mode sobre IPV6 son una mejor alternativa a soluciones similares en IPV4. Como resultado se concluyó que emisiones multicast Sparse mode en competencia por la capacidad del enlace IPV4 presenta un mejor uso del ancho de banda que IPV6. Este artículo sirve de base para mejorar las investigaciones en protocolos multicast para la transmisión de información unidireccional.
\end{abstract}

Palabras clave: Transmisiones multicast, ipv6, ancho de banda, sparse mode.

\begin{abstract}
In the research the design and implementation of the multicast protocol of type Sparse mode was described in order to improve the traffic of data in networks that transport unidirectional information. The design is performed on a network that implements IPV6 as layer 3 protocol of the OSI reference model. To validate the method, the traffic evaluation was performed by analyzing the bandwidth, delays and number of bits sent by the multicast protocol Sparse mode carried out in routers that use IPV6 and IPV4 in single mode or in Dual Stack, for the analysis was used Jperf performing tests with various parameters. A pilot network with 5 routers was implemented using OSPF as a dynamic unicast routing protocol, as a basis for the implementation of multicast Sparse mode. Two wireless links between 4 nodes of the network were also used to know the multicast response in two scenarios. For the validation, 10 transmissions were performed under IPV4, IPV6 (scenario 1), and Dual stack (scenario 2), taking the average data of each transmission and knowing the bandwidth, delays and amount of transmitted data. Carried out in the laboratory CISCO of the Universidad Nacional del Altiplano. As a hypothesis, it is considered that Sparse mode multicast transmissions over IPV6 are a better alternative to similar solutions in IPV4, as a result multicast transmissions are concluded Sparse mode in competition for the capacity of the IPV4 link presents better bandwidth use than IPV6. This article serves as a basis for improving research on multicast protocols for the transmission of unidirectional information.
\end{abstract}

Keywords: Multicast transmissions, ipv6, bandwidth, sparse mode.

Correspondencia al autor:

email: $\mathrm{xxx}$ 


\section{Introducción}

Los servicios de video y contenidos seguirán siendo los líderes en comparación con todas las demás aplicaciones. El video por internet representará el 79\% del tráfico mundial de internet en 2020 frente al $63 \%$ de 2015. El mundo alcanzará los tres millones de minutos de video por internet mensuales en 2020, lo que equivale a cinco millones de años de video al mes, o alrededor de un millón de minutos de video cada segundo. El video HD y el video por internet de ultra alta definición comprenderán el 82\% del tráfico de video por internet en 2020, mucho más que el $53 \%$ de 2015. (CISCO, 2015).

El video bajo demanda en dispositivos móviles es un área clave en el crecimiento de los datos móviles. En octubre de 2014 Youtube afirmó que un 50\% de su tráfico global lo generaban los dispositivos móviles. Un Nuevo informe de Cisco afirma que el video supondrá un $84 \%$ del total del tráfico online en 2018 . El video IP supondrá un 79\% del tráfico global en 2018, frente al 66\% de 2013, mientras que el video en Ultra HD supondrá el 11\% del tráfico de video IP en 2018. (Agile Content, 2016).

El desarrollo de internet se basó fundamentalmente en establecer un proceso abierto. Fundamentalmente, Internet es una "red de redes", cuyo protocolo está diseñado para permitir que las redes puedan interoperar entre ellas. En un principio, estas redes representan diferentes comunidades académicas, gubernamentales y de investigación, cuyos miembros cooperan para desarrollar estándares comunes y gestionar los recursos conjuntos. Más tarde, cuando se comercializó el internet, los vendedores y los operadores se unieron al proceso de desarrollo de protocolo abierto y ayudaron a dar rienda suelta a la era sin precedentes de crecimiento e innovación (ISOC, 2015).

La implementación del Protocol Independent Multicast Sparse Mode sobre IPV6 (IETF, 1998) para mejorar la performance de multimedios en las redes de datos, se lleva a cabo buscando aprovechar las ventajas que esta pueda brindar para permitir mejorar y ampliar el acceso a redes con poca capacidad de ancho de banda.

La evaluación del tráfico cursado en redes que implementan el protocolo PIM-SM sobre IPV6 (CISCO System, 2011), permite analizar las ventajas que ofrece sobre soluciones similares basadas en el protocolo IPV4, su impacto en el ancho de banda, retardos, pérdida de paquetes, y jitter (Trejo). Esta evaluación permite una adecuada configuración y diseño de la arquitectura de red piloto a implementarse, equipos a utilizar, uso de sistemas operativos, configuración de equipos en capa 3 del modelo OSI. (CISCO, 2012).

Para la validación se utilizó Jperf como herramienta para analizar el tráfico sobre una red IPV4 o IPV6 bajo diferentes variables utilizadas en entornos multicast. El objetivo del trabajo de investigación fue evaluar el ancho de banda en transmisiones multicast sparse mode implementadas en redes IPV6 manteniendo la hipótesis de que las emisiones multicast Sparse mode sobre IPV6 son una mejor alternativa en el uso de ancho de banda en soluciones similares con IPV4.

\section{Método}

El trabajo de investigación corresponde al tipo causal-explicativo, mediante la manipulación de la variable independiente para ver el efecto que tiene en la variable dependiente. El tipo es descriptivo porque es necesaria la descripción del uso práctico del conocimiento y teorías del protocolo IP para mejorar y resolver el problema específico llamado emisiones multicast Sparse mode. Debido a que la población es pequeña, un paquete de datos comprimido, el cual es enviado por diez veces consecutivas en la red experimental y en las dos redes de comprobación, se ha considerado todo el universo, en consecuencia, se toma a toda la población.

Los instrumentos son la evaluación, los investigadores y la toma de medidas para la evaluación del comportamiento de la red piloto.

Para la toma de datos se plantea dos escenarios:

- Escenario 1: emisiones multicast Sparse mode en IPV4 e IPV6 de forma individual.

- Escenario 2: emisiones multicast Sparse mode en Dual Stack IPV4 e IPv6.

Plan de Recolección de Datos

- Diseñar la topología de red piloto con equipos reales en el laboratorio CISCO de la Universidad Nacional del Altiplano.

- Realizar la programación de los routers con enrutamiento dinámico unicast OSPF, y enrutamiento multicast para IPV4 e IPV6 (Pronk, 2016).

- Utilizar Jperf a 1mbps en IPV4 e IPV6 para las pruebas. 
- Realizar las pruebas necesarias, escenario 1: emisiones multicast Sparse mode en IPV4 e IPV6; y escenario 2: Dual Stack sobre la red piloto implementada.

Hardware

- Laptop HP Pavilion DV5

- Routers CISCO 2901
Software

- Windows 10

- Jperf

La red piloto implementada utiliza el diseño de la Figura 1.
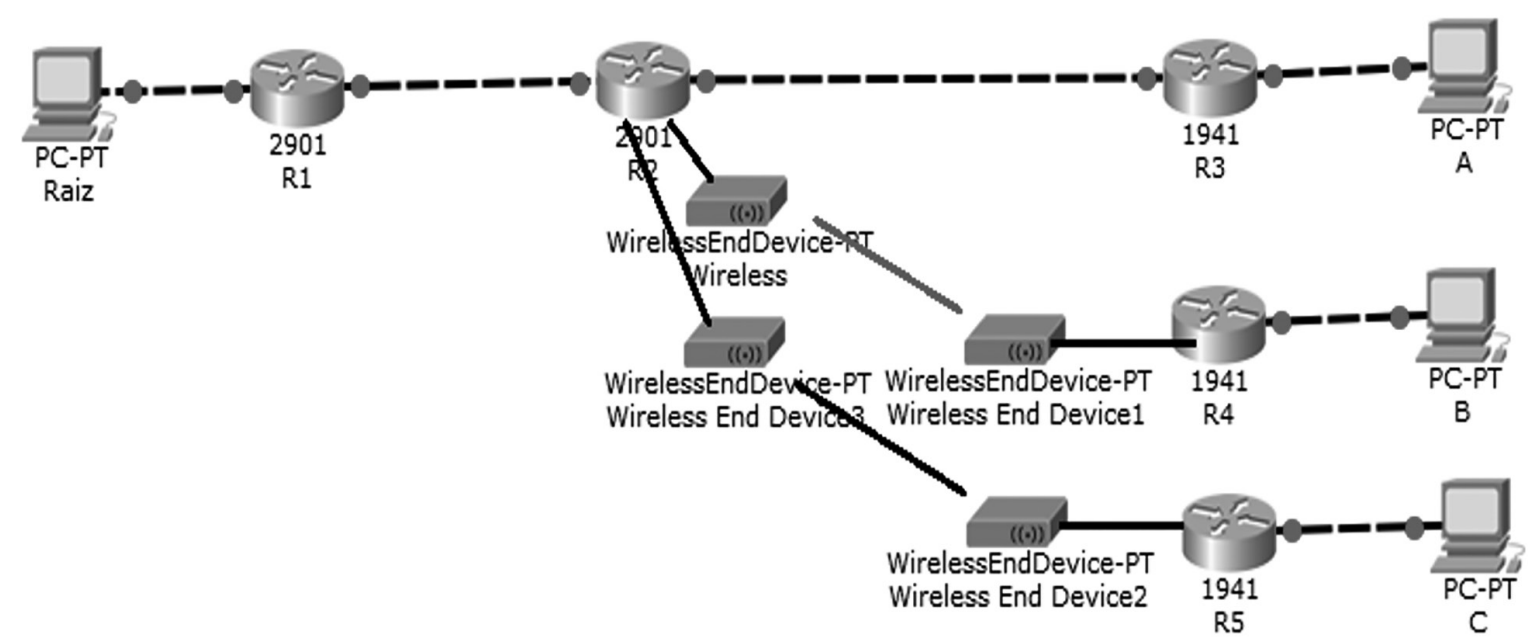

Figura 1. Diagrama topológico de la red

Los materiales utilizados son:

- Laptop

- Modelo

: HP Pavilion

- Procesador

: Pavilion DV5

- Memoria Instalada (RAM)

- Tipo de Sistema

: Intel Core i3-350M a $2,26 \mathrm{GHz}$

- Desktop

$3.00 \mathrm{~GB}$

- Procesador

- Memoria RAM

: Sistema Operativo de 64 bits Windows 10

- Sistema Operativo

: Intel Core i3

: $4 \mathrm{~GB}$

: Sistema Operativo de 64 bits Windows 10

- Routers

: Cisco 2901

- Cisco IOS Software, 2800 Software Versión 15.1(4)M6

- ROM: System Bootstrap, Version 12.4(13r)T

- 2GigabitEthernet interfaces

- 2 Low-speed serial (sync/async) interfaces

- 1 Virtual Private Network (VPN) Module

- DRAM 512MB

- FLASH 256MB

- AP TPLINK TL-WA701ND

- $2.4 \mathrm{GHZ}$

- Antena $5 \mathrm{dbi}$

- $802.11 \mathrm{~b} / \mathrm{g}$

Los parámetros mencionados inciden en el ancho de banda ofrecido por la red piloto a utilizarse en los dos escenarios a analizar. 
Software:

- Sistema Operativo de 64 bits Windows 10

- Herramienta de análisis de tráfico Jperf

- Software de simulación de redes: Packet Tracert V 7

- Microsoft Excel Versión: 2013

\section{Resultados}

\section{Multicast IPV4 e IPv6 individuales}

Para la investigación en este primer escenario se emitirá tráfico multicast IPV4 individualmente durante 300 segundos y se repetirá el proceso 5 veces, tomando el promedio en cada repetición. Se realizará el mismo procedimiento sobre una red IPV6 individual.

Utilizando IPV4 e IPV6 individualmente el programa Jperf se configuró con los siguientes parámetros para IPV4: protocolo UDP, dirección de grupo multicast 239.255.255.250, BW 1Mbps, puerto 5001, con un intervalo de 300 segundos. Esto se muestra en la figura 2.

El comando de configuración en el servidor es: bin/ iperf.exe -s -u -P 0 -i 300 -p 5001 -B 239.255.255.250 $-\mathrm{fk}$

El comando de configuración en el cliente es: bin/ iperf.exe -c 239.255.255.250 -u -P 1 -i 300 -p 5001 -B 239.255.255.250 -f k -b 1.0M -t 10 -T 15

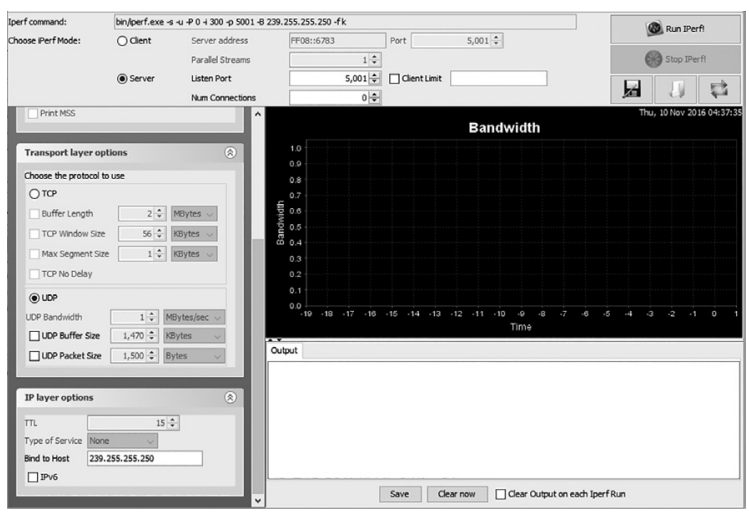

Figura 2. Jperf IPV4

La configuración de IPV6 en Jperf utilizó los siguientes parámetros: protocolo UDP, dirección de grupo multicast FF08::6783, BW 1Mbps, puerto 5001, con un intervalo de 300 segundos. Esto se muestra en la figura 3. El comando de configuración en el servidor es: bin/ iperf.exe -s -u -P 0 -i 300 -p 5001 -B FF08::6783 -V -f $\mathrm{k}$. El comando de configuración en el cliente es: bin/iperf.exe -c FF08::6783 -u -P 1 -i 300 -p 5001 -B 239.255.255.250 -f k -b 1.0M -t 10 -T 15

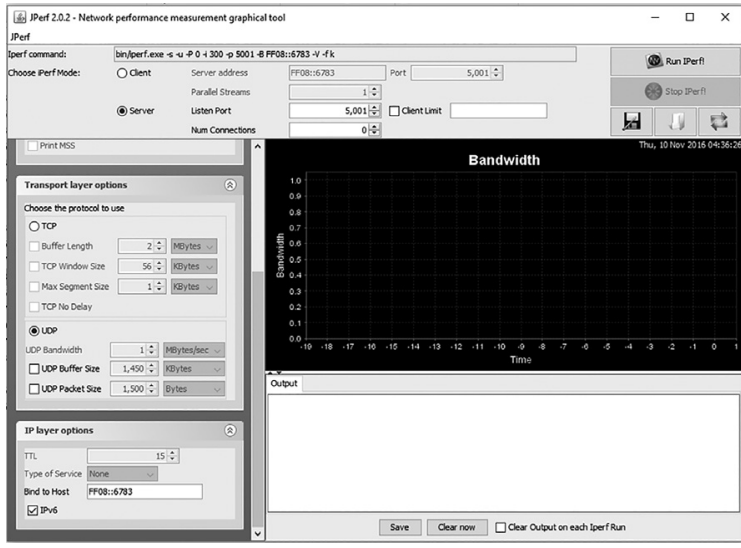

Figura 3. Jperf IPV6

Se obtuvo 3 resultados en el nodo raíz y los nodos finales A (Enlace $1 \mathrm{~Gb}), \mathrm{B}($ Enlace $1 \mathrm{Mb})$ y C (Enlace $2 \mathrm{Mb}$ ). En la figura 4 se observa el ancho de banda requerido por el emisor en IPv4 e IPv6 al ser un enlace con capacidad de 1 Gbps. En ambas emisiones satisface la capacidad exigida por el emisor.

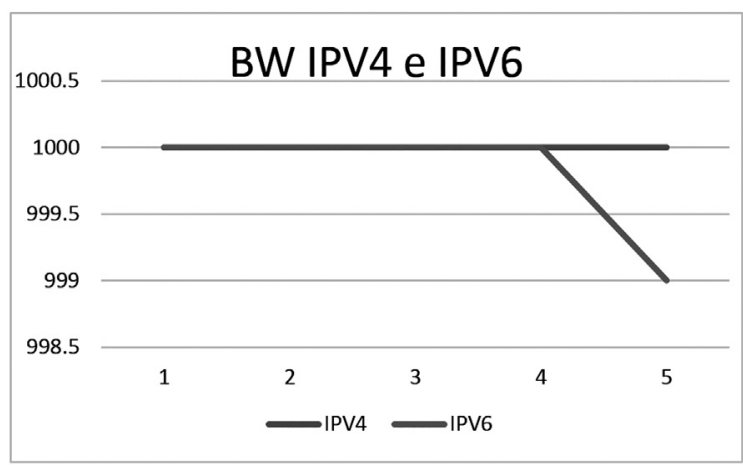

Figura 4. Ancho de banda en el nodo raíz

En la figura 5, el ancho de banda en el nodo A en IPv4 e IPv6, soporta el tráfico enviado por el emisor al tener una capacidad de $1 \mathrm{Gbps}$.

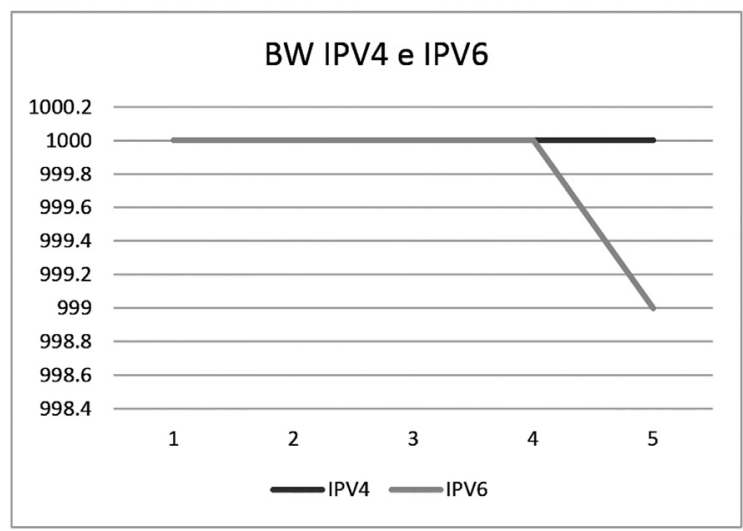

Figura 5. Ancho de banda en el nodo A 
En la figura 6, el ancho de banda en el nodo B, en IPv4 e IPv6, al tener una capacidad de 1000kbps y ser exigido al $100 \%$, el ancho de banda que soportó en IPv6 fue de 895kbps y de $975 \mathrm{Kbps}$ en IPv4.

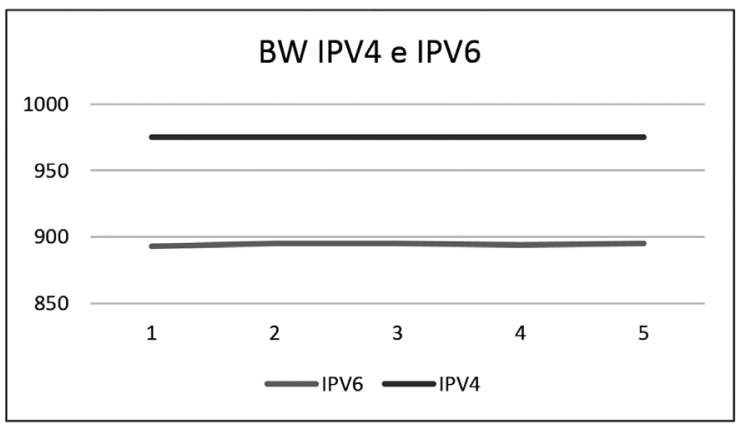

Figura 6. Ancho de banda en el nodo B

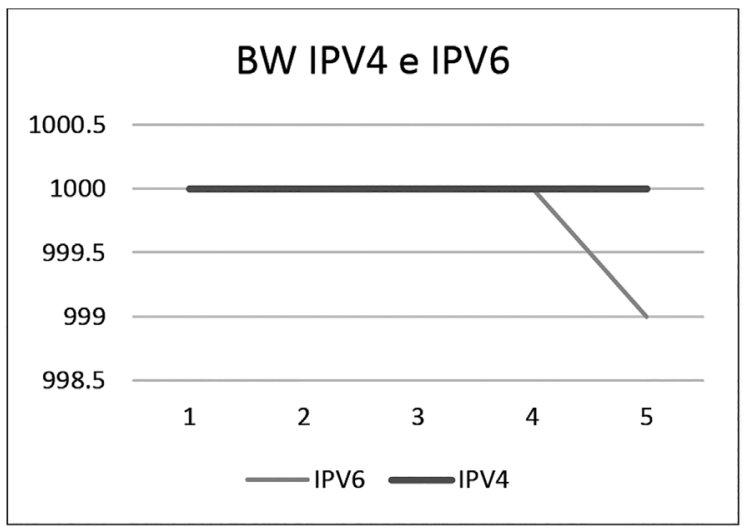

Figura 7. Ancho de banda en el nodo C

En la figura 7, el ancho de banda en el nodo $\mathrm{C}$ en IPv4 e IPv6, soporta el tráfico enviado por el emisor al tener una capacidad de $2 \mathrm{Mbps}$.

En la siguiente tabla se muestra el promedio de cada nodo:

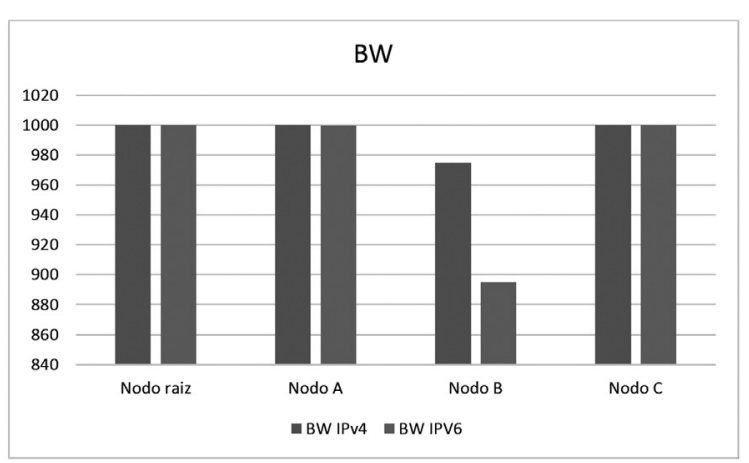

Figura 8. Valor Promedio IPV4 e IPV6 individuales
Se observa en la figura 8 que en transmisiones multicast Sparse mode el ancho de banda en el nodo raíz se mantiene a pesar de tener 3 conexiones simultáneas de los nodos A, B y C.

El nodo A, al ser un enlace de $1 \mathrm{Gbps}$, mantiene las mismas características que el nodo raíz.

El nodo B al ser un enlace de 1Mbps IPV4 utiliza un ancho de banda de $975 \mathrm{kbps}$ e IPV6. Utiliza 895Kbps, pero se debe considerar que IPV4 presenta una pérdida de paquetes en promedio del $1.8 \%$ e IPV6 presenta una pérdida de paquetes del $10 \%$.

El nodo $\mathrm{C}$ al ser un enlace de $2 \mathrm{Mbps}$, el doble del ancho de banda raíz, mantiene las mismas características del ancho de banda en el nodo raíz.

\section{Dual stack}

Se utiliza los mismos parámetros que las transmisiones individuales, diferenciando que las transmisiones en dual stack se realizan en simultáneo IPV4 e IPV6, realizándose 10 pruebas.

En la figura 9 se observa el ancho de banda requerido por el emisor en IPv4 e IPv6 en modo dual stack, al ser un enlace con capacidad de $1 \mathrm{Gbps}$. En ambas emisiones satisface la capacidad exigida por el emisor.

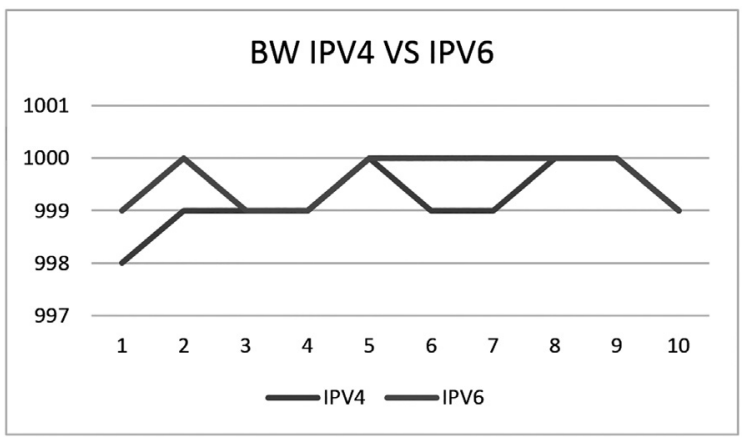

Figura 9. Ancho de banda en el nodo raíz

En la figura 10, el ancho de banda en el nodo A en IPv4 e IPv6, soporta el tráfico enviado por el emisor al tener una capacidad de $1 \mathrm{Gbps}$. Se observa que no existe pérdida de paquetes. 




Figura 10. Ancho de banda en el nodo A

En la figura 11, el ancho de banda en el nodo B, en IPv4 e IPv6, debido a la competencia por el enlace de los dos protocolos, IPV4 tiene mejores resultados al transmitir $423 \mathrm{Kbps}$, respecto a $345 \mathrm{Kbps}$ en IPv6.

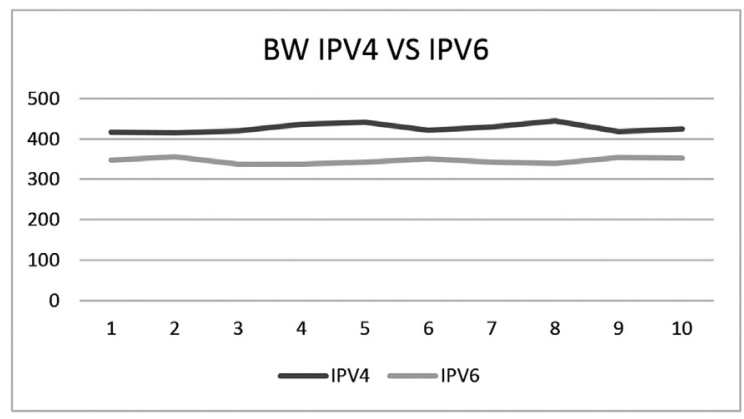

Figura 11. Ancho de banda en el nodo B

En la figura 12, el ancho de banda en el nodo C es similar al nodo B. Debido a la competencia por el enlace de los dos protocolos, IPV4 trasmitió 972Kbps respecto a $777 \mathrm{Kbps}$ en IPv6

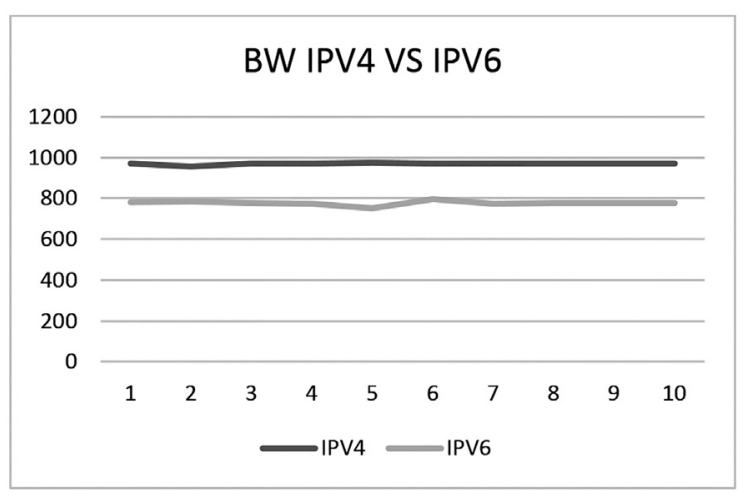

Figura 12. Ancho de banda en el nodo C

En la figura 13 se muestra el promedio en cada nodo. Se observa en la figura 13 que en transmisiones multicast Sparse mode dual stack, el ancho de banda en el nodo raíz se mantiene a pesar de tener 6 conexiones simultáneas 3 de IPV4 y 3 de IPV6, de los nodos A, B y C.

El nodo A, al ser un enlace de $1 \mathrm{Gbps}$, mantiene las mismas características que el nodo raíz.

El nodo B, al ser un enlace de 1Mbps IPV4, utiliza un ancho de banda de $423 \mathrm{kbps}$ e IPV6 utiliza $345 \mathrm{Kbps}$, pero se debe considerar que IPV4 presenta una pérdida de paquetes en promedio del 57\% e IPV6 presenta una pérdida de paquetes del $65 \%$.

El nodo $\mathrm{C}$, al ser un enlace de $2 \mathrm{Mbps}$, utiliza un ancho de banda de $972 \mathrm{kbps}$ e IPV6 utiliza $777 \mathrm{Kbps}$, pero se debe considerar que IPV4 presenta una pérdida de paquetes en promedio del $2.5 \%$ e IPV6 presenta una pérdida de paquetes del $22 \%$.

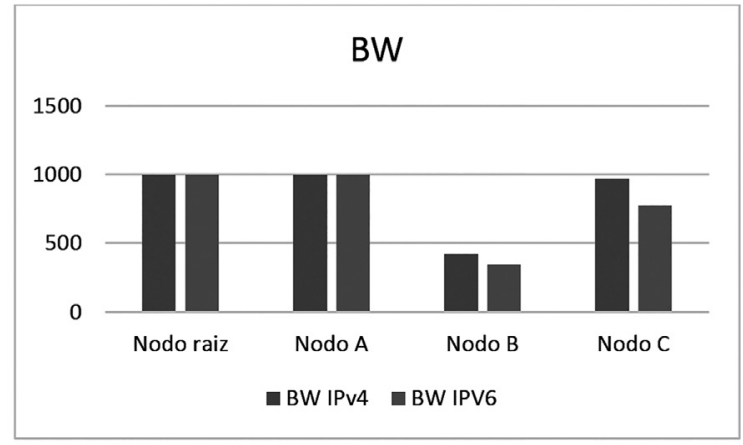

Figura 13. Valor promedio IPV4 e IPV6 individuales

\section{Conclusiones}

Se utilizó el software Jperf para analizar el ancho de banda y obtener valores promedio para un análisis y medida de emisiones multicast sparse mode, en condiciones ideales y de saturación del enlace. De los resultados obtenidos en redes IPV4 e IPV6 individuales, si el flujo multicast atraviesa un enlace con un ancho de banda superior al emitido, se mantienen los datos y ancho de banda originales. Si atraviesa un enlace que tiene un ancho de banda idéntico o menor al ancho de banda a emitirse, IPV6 presentará mayores pérdidas de paquetes al utilizar un ancho de banda menor al de IPV4. Por lo cual se recomienda utilizar IPV4 en condiciones de competencia por el enlace.

En redes Dual Stack, los resultados son similares a la transmisión de IPV4 e IPV6 individualmente, con la diferencia que en enlaces con anchos de banda similares al emitido, la pérdida de paquetes es mucho mayor que el $50 \%$. Por lo cual, no se recomienda utilizarlo en condiciones con reducido ancho de banda.

Al finalizar el trabajo de investigación se concluye que PIM-SM sobre redes IPV6 tiene un mejor desen- 
volvimiento que implementaciones similares en redes IPV4, en medios de transmisión con velocidades de transmisión adecuadas.

\section{Referencias}

Agile Content (2016). Discovery meeting (Agile Content). Recuperado de http://www.agilecontent.com/ documents/es/facts/AGIL.HR.Foro+MedCap+31. may.2016.pdf

CISCO (1 de agosto de 2012). Implementing IPv6 Multicast (CISCO). Recuperado el 1 de febrero de 2016, de http://www.cisco.com/c/en/us/td/docs/ios-xml/ios/ ipv6/configuration/xe-3s/ipv6-xe-36s-book/ip6-multicast.html

CISCO (2015). White paper: Cisco VNI Forecast and Methodology, 2015-2020. Obtenido de Service Provider Forecasts and Trends. Recuperado de http://www. cisco.com/c/en/us/solutions/collateral/service-provi- der/visual-networking-index-vni/complete-white-paper-c11-481360.html

CISCO System (2011). IPV6 Configuration Guide, CISCO IOS. San José: CISCO Press.

IETF (Julio de 1998). IP Versión 6 Addressing Architecture (IETF). Recuperado el 20 de febrero de 2016, de https://tools.ietf.org/html/rfc2373

ISOC (5 de junio de 2015). The Internet and Sustainable Development (ISCO). Recuperado el 2 de noviembre de 2016, de http://www.internetsociety.org/doc/internet-and-sustainable-development

Pronk, R. (2016). Impact of IPV6 on WiFi based netowrks. Enschede: UNIVERSITY OF TWENTE.

Trejo, N. B. (s.f.). Aplicación Multicast IPV6 seguro a servicios de información en entornos grid. Madrid: Universidad Complutense de Madrid. 